\title{
Facilitating protein denaturation in organic solvent and the contribution to the promoting dispersion of graphite nanoplatelets in a polymer
}

\author{
T. Liu, Y. Wang, A. Eyler, Y. C. Chang, W. H. Zhong* \\ School of Mechanical and Materials Engineering Washington State University, WA 99164 Pullmanm, USA
}

Received 9 January 2015; accepted in revised form 19 February 2015

\begin{abstract}
Denatured proteins, natural macromolecules are very attractive for advanced nanocomposites owing to their multiple functional chemical groups. However, denaturation processes were only successfully conducted in an aqueous environment, limiting their broad applications in hydrophobic polymers. In this study, we report an effective approach of denaturing soy protein at nanoscale in an organic solvent. Further, the denatured soy protein was found to be able to infiltrate between the graphite nanoplatelet (GNP) layers to reduce the thickness of GNPs and improve the dispersion of the nanoparticles in either the suspension or the final polymeric nanocomposites. As a result, remarkable improvements in transparency and electrical conductivity have been achieved for the nanocomposites with the GNPs treated by the denatured soy protein.
\end{abstract}

Keywords: nanocomposites, soy protein, denaturation, biotreatment of nanofillers, organic solvent

\section{Introduction}

It is well known that, to create high-performance polymeric nanocomposites, dispersion of the nanofillers in the polymer matrix, and interfacial interactions between nanofillers and matrix are the two critical factors. Meanwhile, carbon nanofillers, including nanofibers (CNF), nanotubes (CNT), and graphitic nanoplatelets (GNP) are the most popular nanofillers employed to make polymeric nanocomposites $[1,2]$. In order to improve the dispersion and interfacial interactions, numerous chemical approaches to the surface modification for carbon nanofillers have been intensely studied [3-8]. It is noted that chemical approaches are often performed via complicated processing procedures, even involving toxic chemicals.

Compared with the various chemical methods, biotreatment provides a more significant avenue to the treatment of nanofillers in an environmentally friendly, simple and effective way. For the biotreatments such biomolecules as polysaccharides [9], albumin [10], enzymes [11], and deoxyribonucleic acid (DNA) [12], can be either covalently or noncovalently performed for the nanofillers. Proteins are full of polar groups such as amino acids constructing the polypeptide causing a variety of interactions and have been of great interest for making polymeric composites [13]. However, another significant application of them that has not been paid adequate attention is that they can be used as a 'green' functional agent to treat nanoparticles for effectively improving dispersion of nanoparticles thus improving the properties of the nanocomposites based on their various functional groups in protein structures. Strategies for harnessing these unique structures and functional groups are attractive for

\footnotetext{
${ }^{*}$ Corresponding author, e-mail: katie zhong@wsu.edu

(c) BME-PT
} 
fabrication of high-performance nanocomposites $[14,15]$.

Recently, soy protein, an abundant plant protein, showed great potential for application into biological functionalization of nanofillers [16-21]. The availability of various groups (such as amines, hydroxyls, etc.) along the soy protein molecules provides possibilities for modification of nanofillers via strong interactions, such as hydrogen bonding or even chemical bonding [18]. However, it should be pointed out that pristine soy proteins exist as big particles (around $30 \mu \mathrm{m}$ [13]) united by strong internal interactions and cannot be directly used for the biotreatment. An effective way to break the native interactions and disentangle the soy protein structures for biotreatment is to effectively denature the pristine soy protein. To this end, aqueous solution with salts is usually used. At the same time, the polymer matrix for the composite has to be water-soluble in order to homogeneously mix the bio-treated nanofillers and the polymer solution. However, most of the polymers cannot be dissolved by aqueous solvents, which indicates that the biotreatment of nanofillers by denatured soy protein for polymeric nanocomposites can't be realized in aqueous systems. Thus far, biotreatment of nanofillers in nonaqueous environment, that is, in organic solvent, which has not been reported, will be critical for soy protein to be used to develop various nanocomposites for wide applications.

In this study, for the first time, we report a method that leads the pristine soy protein isolate (SPI) to be effectively denatured in a non-aqueous environment via the help of a denaturing agent, cetyl alcohol (CA). The biotreatment of nanofillers (GNP, for example) through use of the denatured SPI was also carried out in organic solvent, chloroform. The effects of the biotreatment on exfoliation and dispersion of graphite were characterized via confocal microscope and field emission scanning electron microscopy. The optical transmittance and electrical properties of the resulting polymeric nanocomposites were investigated through UV-visible spectrophotometer and Dielectric Analyzer, respectively. The results indicate that the denatured SPI can remarkably improve the GNP exfoliation and dispersion in the final nanocomposites. Compared with the sample without the biotreatment, the optical transmittance of the resulting nanocomposites with $1 \mathrm{wt} \%$ of GNPs is increased by 3.2 times, and the electrical conductivity increases by 11 orders of magnitude (at $10^{-2} \mathrm{~Hz}$ ).

\section{Experimental section \\ 2.1. Materials}

Granule-shaped polycarbonate (PC, Lexan 101) as the matrix used in this study was kindly provided by SABIC Innovation Plastics Company, MA, USA. The graphitic nanoplatelets (GNPs) obtained from XG Science Inc., MI, USA, were pre-exfoliated with an average diameter of $\sim 5 \mu \mathrm{m}$ and $\sim 5 \mathrm{~nm}$ in thickness. Soy protein isolate (SPI) was donated by ADM Co., IL, USA, and contained $4.8 \%$ moisture and $90.9 \%$ dry basis protein. Chloroform, obtained from Fisher Scientific, PA, USA, was used as the solvent for both PC and GNPs surface modification with SPI. Cetyl alcohol (1-Hexadecanol, 98\%) as SPI denaturing agent was purchased from Alfa Aesar, MA, USA.

\subsection{Preparation of nanocomposite films}

The bio-modification of GNPs was accomplished by dispersing pristine GNPs $(0.02 \mathrm{~g})$ and SPI $(0.02 \mathrm{~g})$ in equal amounts in chloroform $(5 \mathrm{~mL})$ via $15 \mathrm{~min}$ high power probe ultrasonication (Branson 450). In order to assist SPI denaturation in chloroform, $0.05 \mathrm{M}$ of cetyl alcohol (CA) was applied as surfactant. The mixture was then heated with oil-bath at $65^{\circ} \mathrm{C}$ for $4 \mathrm{~h}$. The preparation procedure for the composite membranes starts by completely dissolving PC $(2 \mathrm{~g})$ in chloroform $(10 \mathrm{mLl})$ via spin mixing. Simultaneously, the pristine GNPs or SPI-treated GNPs with and without CA were mixed with PC-chloroform solution using room temperature bath-sonication for $10 \mathrm{~min}$ to obtain homogenous suspension. Finally, an aluminum mold was used to cast the composite solution on glass substrate. The thickness of all films was $0.04 \mathrm{~mm}$. After completely evaporating the solvent, the composite film was taken off and dried at $50^{\circ} \mathrm{C}$ overnight to remove any moisture. Table 1 presents the descriptions of each composite film prepared in this study.

Table 1. Detailed description for each nanocomposite

\begin{tabular}{|l|l|c|}
\hline \multicolumn{1}{|c|}{ Composite } & \multicolumn{1}{c|}{ Reinforcement } & SPI denaturation agent \\
\hline PC/GNP & Pristine GNPs & - \\
\hline PC/GNP-CA & Pristine GNPs & - \\
\hline PC/s-GNP & SPI treated GNPs & - \\
\hline PC/s-GNP-CA & SPI treated GNPs & Cetyl alcohol \\
\hline
\end{tabular}




\subsection{Characterizations}

Confocal images were acquired using a ZEISS confocal microscope. The $488 \mathrm{~nm}$ line from an argon laser was used for excitation, and fluorescence emission was detected using a $510 \mathrm{~nm}$ band-pass filter. Images were recorded using a water immersion objective. Optical micrographs, imaged by Olympus BX51, were obtained of thin composite films. Field emission scanning electron microscopy (FESEM) images were taken using a FEI Quanta 200F. Both GNP particles and fracture surface of the composites was prepared to take SEM images. All composite samples were frozen in liquid nitrogen for $5 \mathrm{~min}$ prior to fracturing, and then sputter-coated with gold for electrical conductivity. Alternating current (AC) conductivity was obtained at ambient conditions using a Novocontrol Technologies Alpha$\mathrm{N}$ high resolution Dielectric Analyzer. The sample thickness of the five nanocomposites (Table 1) was prepared for AC conductivity testing, which is ca. $200 \mu \mathrm{m}$. The difference in transparency of the nanocomposite film was described via UV-vis spectroscopy. The UV-visible spectrum was recorded on a Nicolet Evolution 300 UV-visible spectrophotometer (Thermo Fisher Scientific Inc., USA) using a quartz cell in the wavelength range of 200-700 $\mathrm{nm}$.

\section{Results and discussion}

Denaturation of SPI is the first step for the biotreatment. The study on the realization of the denaturation of SPI in organic solvent is very rare. As one of the results from denaturation, the SPI particle size will change a lot after the denaturation. Since SPI can show green fluorescence under the illuminating light (excitation wavelength: $488 \mathrm{~nm}$; emission wavelength: $510-530 \mathrm{~nm}$ ), one can identify the change in particle size by confocal images obtained from confocal microscope. Figure $1 \mathrm{a}$ and $1 \mathrm{~b}$ are the confocal images of the SPI particles after thermal treatment in chloroform without and with the addition of denaturing agent, cetyl alcohol (CA), respectively. By comparing the confocal images, one can find that the addition of CA can remarkably reduce the size of SPI, indicating that the SPI particles were well-denatured via the help of CA. At the same time, the distinct difference in particle size between poorly denatured and well-denatured SPI is further demonstrated by the laser scattering results as shown by the inserts in Figure 1a and 1b. Clearly, the well-denatured SPI with CA gives rise to a much more trans- parent solution as compared with the poorly denatured SPI without the addition of CA. In addition to the above results in the solvent, the solid SPI particles were also characterized by SEM images. As shown in Figure 1c up and down, there is a big difference in the particle size for the poorly denatured (up) and well-denatured SPI particles (down). For the well-denatured protein, the SPI particles become nanoparticles (ca. $70 \mathrm{~nm}$ ); while, the other one almost remains the same size as the pristine SPI (ca. $30 \mu \mathrm{m})$. Besides, Circular Dichroism (CD) technique was also applied to characterize the change in protein structures for the two samples. Ellipticity, defined as a differential absorbance between right-hand-polarized and left-hand-polarized light through a protein, is a common parameter to characterize the protein structures. For the structure analysis of protein, the wavelength of 190-250 $\mathrm{nm}$ can provide the information of secondary structures, including $\alpha$-helix, $\beta$-sheet, and random coil. In comparison of the ellipticity at $220 \mathrm{~nm}$ in Figure 1d, which corresponds to an $\alpha$-helical secondary structure, the lower ellipticity of the denatured SPI solution (green) indicates that the well-denatured SPI possesses unfolded and more randomized structures.

Since proteins are famous for their abundance in functional groups and structures, the denatured SPI with open structures should be promising for biotreatment of nanoparticles, such as GNPs. Figure 2 shows the FESEM and TEM images of the GNP samples treated by SPI with the two different denaturation states as described above. For the GNP sample treated by poorly denatured SPI (called as $s$ GNP), no notable interaction was detected by the SEM images as shown in Figure 2a and $2 b$. At the same time, lots of un-denatured SPI particles can be found as indicated in Figure 2a. Further, the bare surface of the GNP sheet (Figure 2b) and numerous graphene sheets overlapped together (insert in Figure $2 b$ ) indicate that the poorly denatured SPI cannot give rise to meaningful interaction with other nanoparticles. Additionally, the $s$-GNP suspension in chloroform was found to be precipitated in a few hours, as shown in the insert in Figure 2a. In comparison, significant interaction between the well-denatured SPI and GNP was confirmed. Firstly, it was difficult to find big protein particles in the GNP sample treated by the well-denatured SPI (called as $s$-GNP-CA) as shown in Figure 2c. Also, it was found that the denatured SPI nanoparticles can coat the 


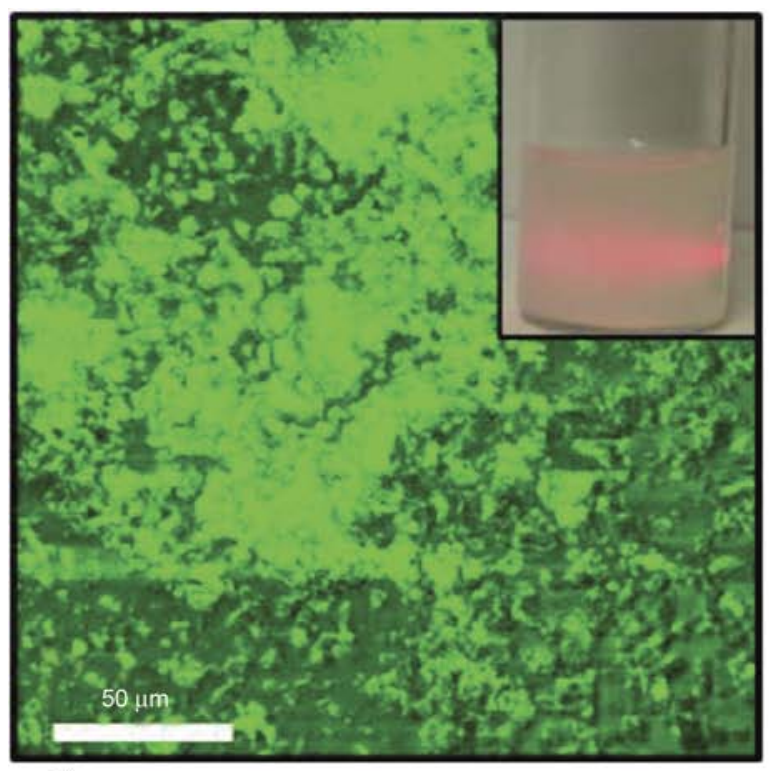

a)

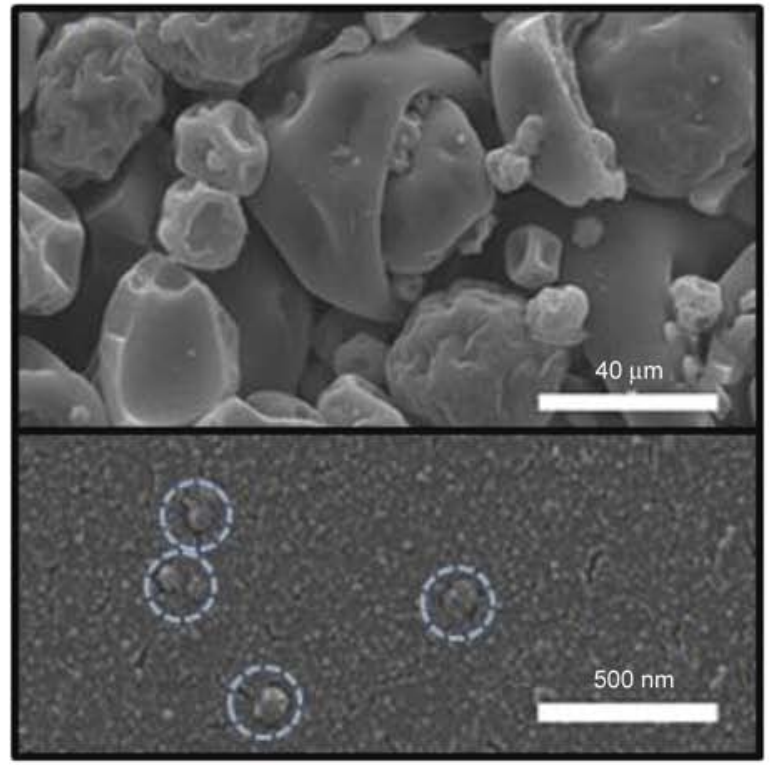

c)

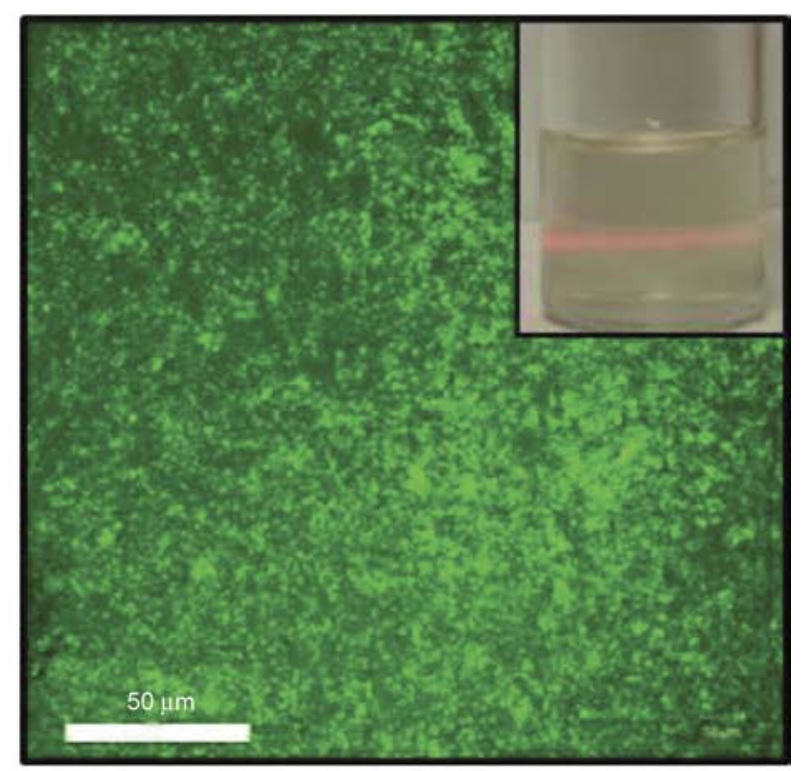

b)

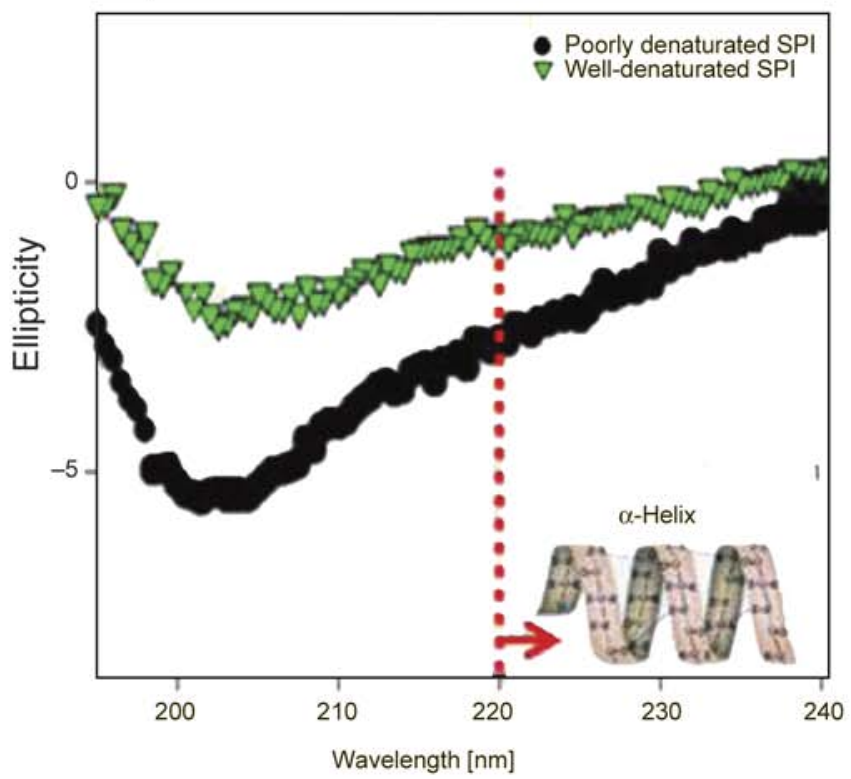

d)

Figure 1. (a) and (b), confocal images of the poorly and well-denatured SPI particles in chloroform, respectively; the inserts are the laser scattering result for the corresponding suspension; (c) up and down, SEM images of the poorly and well-denatured SPI particles, respectively; (d) circular dichroism results for the two samples with different denaturation states

GNP surface very well as shown by the SEM image at high magnification (Figure 2d), which indicates a strong interaction between the denatured SPI and GNP. As a benefit from the strong interaction, a stable $s$-GNP-CA suspension was formed and can maintain for even several weeks (insert in Figure 2c). Finally, compared the TEM image of s-GNP (insert in Figure 2b), the improved transparency of GNP sheet can be found in $s$-GNP-CA (insert in Figure 2d), revealing that the thickness of GNP was reduced. These findings indicate that denatured SPI can be a very effective biotreatment agent for GNPs since nanoscale SPI particles will provide much larger active surface area as well as more functional groups to interact with nanofillers. Although it needs further studies to learn the specific interactions, the variety of functional groups and interactions along the denatured protein chain should be the key for the SPI coating on the surface of GNPs. For example, molecular modeling results have shown that denatured protein may change its chain configuration accordingly to generate good interaction with GNPs [22]. Further, there are also residual groups on GNPs [23], which can provide extra spots for the interac- 


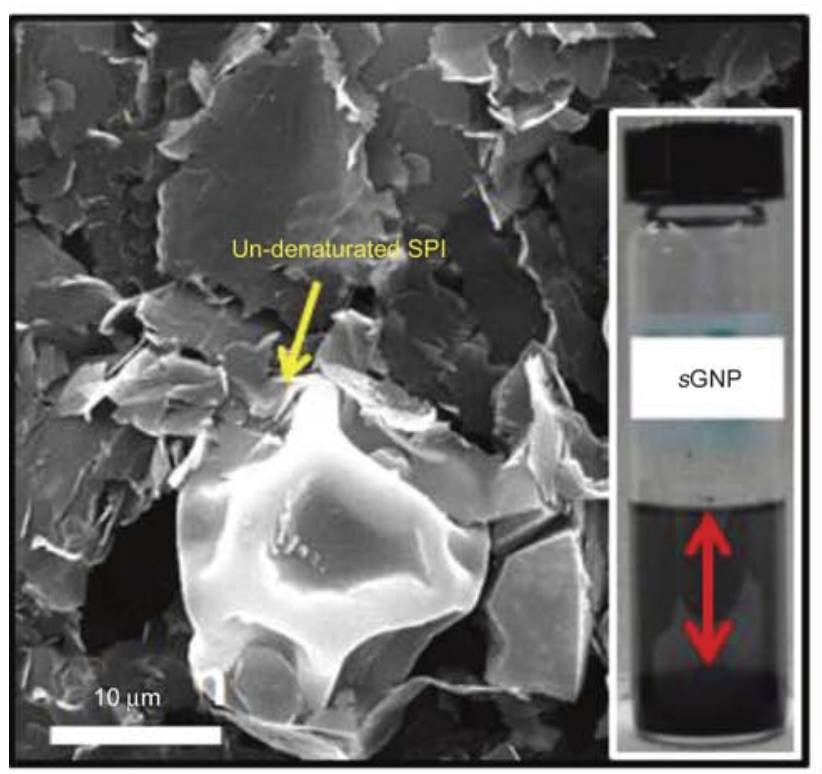

a)

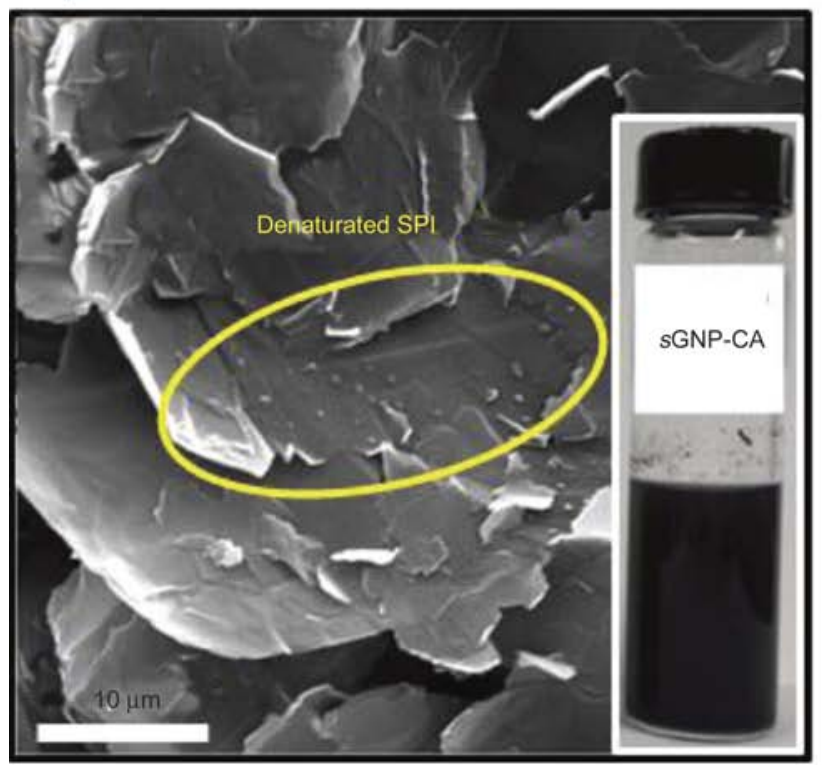

c)

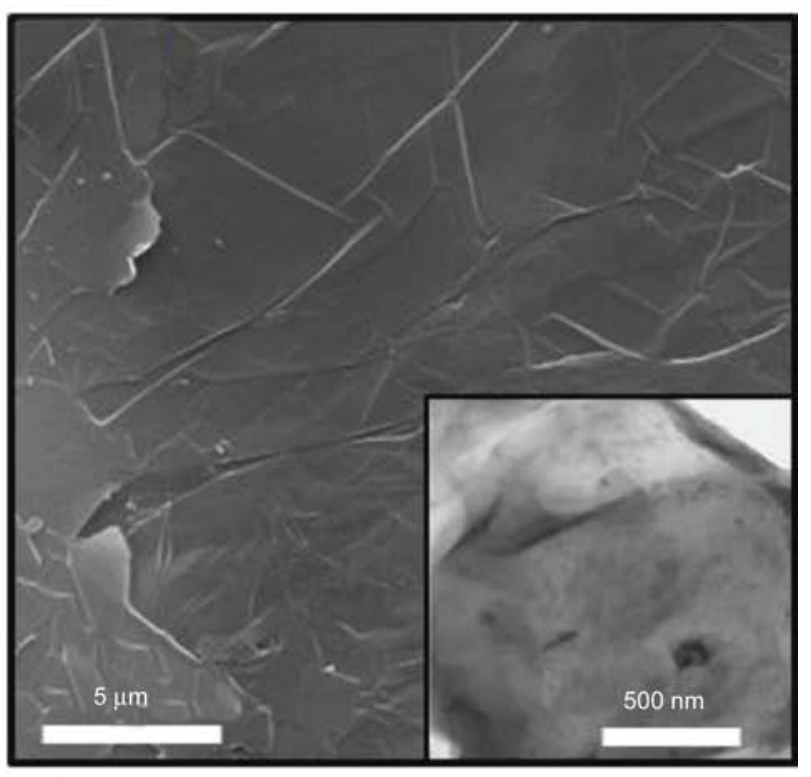

b)

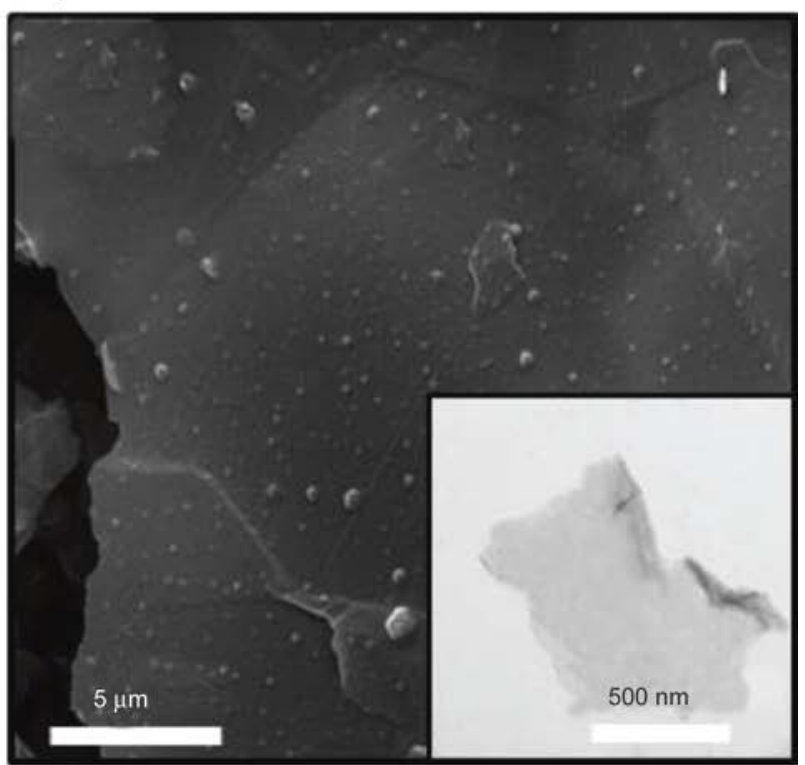

d)

Figure 2. FESEM images of the GNP samples treated by the SPI samples: (a \& b) treated by the poorly denatured SPI, (c \& d) treated by the well-denatured SPI. Inserts in (a) and (c) are digital photos for the corresponding suspensions in chloroform, inserts in (b) and (d) are the corresponding TEM images for the treated GNPs.

tions with denatured protein. Based on Figure 2a$2 \mathrm{~d}$, one can conclude that the well-denatured SPI can generate strong interactions with other nanoparticles, such as GNPs.

To summarize the above results, one can conclude that, the denaturing agent, $\mathrm{CA}$, is critical for the biotreatment of GNP via SPI. Firstly, it helps the denaturation of SPI in the organic solvent, chloroform, and reduces the SPI particle size into nanoscale. The polar group, hydroxyl, of CA should be one key for the SPI denaturation. It may form hydrogen bond with the possible polar groups on SPI and destroy the built-in interactions inside the pristine SPI parti- cles in the organic solvent. Further, the unfolded SPI particles, that is, the denatured SPI particles provide various functional groups available for interactions with nanofillers [24]. Finally, the denatured SPI particles can coat the nanoparticle surface via some strong interactions, enabling the biotreatment of nanoparticles in an organic environment. Figure 3 further illustrates this biotreatment process via denatured SPI as described above.

As one significant application of the bio-treatment, we further studied the polymeric nanocomposites based on the $s$-GNP-CA. At the same time, other GNP samples were also studied for comparison. 

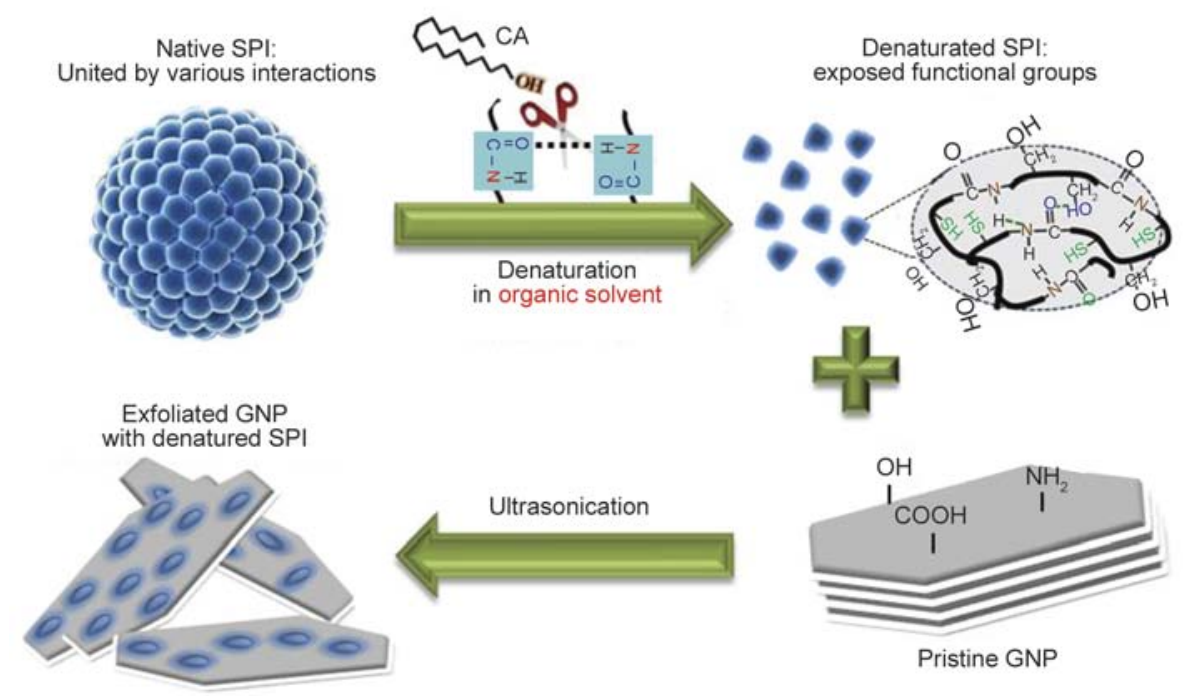

Figure 3. Schematic representation of the biotreatment of GNPs by the denatured SPI; the SPI particles can coat the surface of GNPs through the exposed functional groups on denatured SPI and the available polar groups on GNP [24]

Firstly, the dispersion quality of GNPs in the final nanocomposites has been investigated. The FESEM images for the dispersion of GNPs in the final nanocomposites are shown in Figure $4 \mathrm{a}-4 \mathrm{~d}$. It can be found that lots of GNP agglomerates or pristine GNP particles can be observed for the samples without the biotreatment by the denatured SPI (see Figure 4a4c). For these samples, besides the observation of obvious gaps between the GNP and the polymer matrix (polycarbonate, PC), some GNP layers were pulled out during the breaking, indicating poor interaction between the filler and the matrix. On the contrary, a much more homogenous dispersion of GNP was observed in Figure 4d for the sample with $s$ GNP-CA. Moreover, one can observe very thin GNP particles, few layers of graphene, and no gap between the PC matrix and $s$-GNP-CA can be found, indicating the biotreatment by the denatured SPI can contribute to some strong interactions between the polymer matrix and the GNP filler. These strong interac-

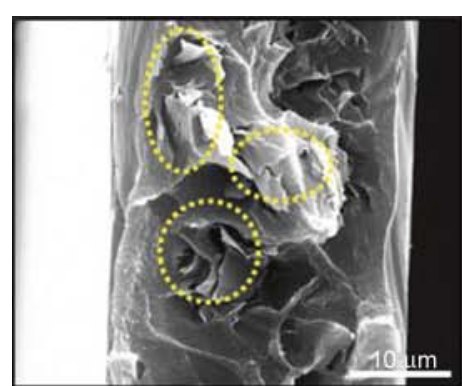

a)

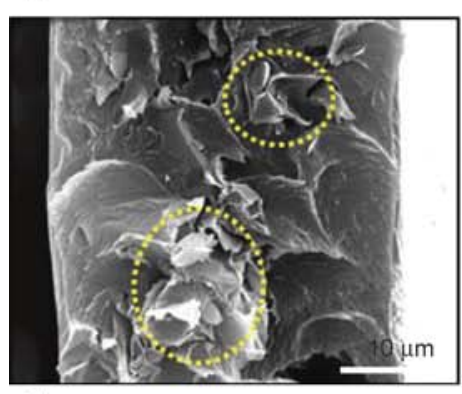

c)

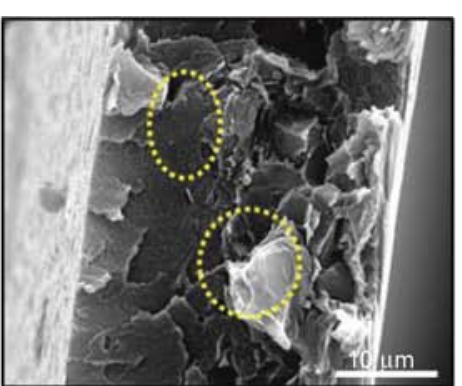

b)

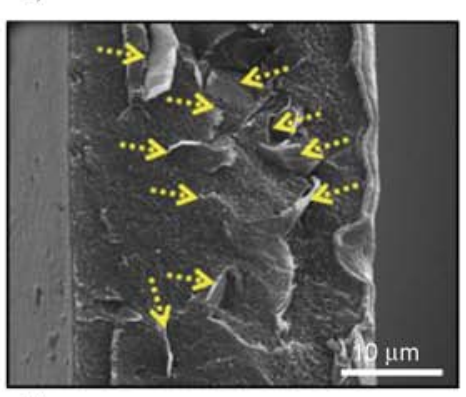

d)

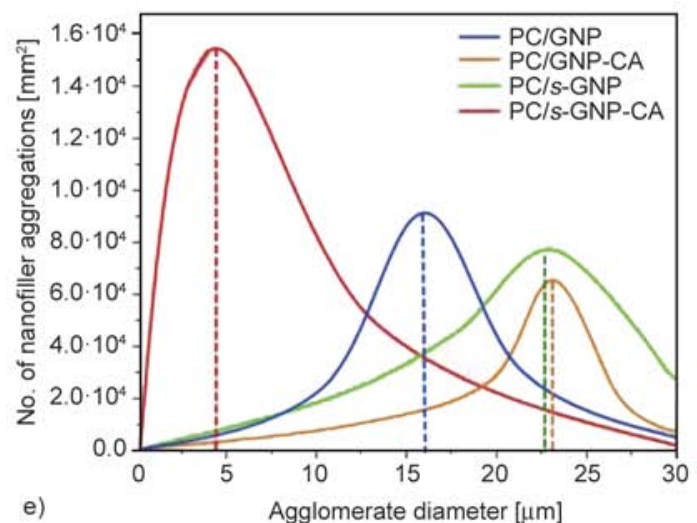

e)

Figure 4. Comparison of the nanofiller dispersion in the nanocomposites with different GNPs. (a-d), SEM images of the fracture surface from nanocomposites with: (a) untreated GNPs, (b) CA treated GNPs, (c) $s$-GNPs, and (d) $s$ GNPs-CA (Scale bars: $10 \mu \mathrm{m}$ ). (e): quantitative analysis of nanofillers dispersion for all the nanocomposites, agglomerates in the size range below $30 \mu \mathrm{m}$. The GNP loading is $1 \mathrm{wt} \%$ for all nanocomposites. 
tions finally give rise to better dispersion and even reduce the thickness of GNPs in the polymer matrix. In addition, the statistical analysis on nanofiller dispersion within the nanocomposites was performed via an in-house prepared MATLAB script, the software developed in our lab [25]. Figure 4e provides the size distributions of GNP aggregates in a range from several nanometers to 30 microns. The proportion of nanofiller aggregation with the size above $15 \mu \mathrm{m}$ in PC/GNP, PC/GNP-CA, and PC/s-GNP are much higher than the nanocomposite with $s$-GNPCA. In the size range below $10 \mu \mathrm{m}$, the contribution of the denatured SPI to the dispersion of GNP is more obvious as the PC/s-GNP-CA sample show the lowest level of filler aggregates, and the number density of aggregates decreased dramatically as the aggregate size increased.

The optical and electrical properties of the nanocomposites were also studied to demonstrate the advantages of the bio-treatment via denatured SPI. The transmittance spectra of all the above four nanocomposite films with thickness of ca. $40 \mu \mathrm{m}$ are presented in Figure 5a. In specific, the transmittance, for example at $600 \mathrm{~nm}$, is about only $19 \%$ for the composite film with the pristine GNP, which is almost opaque as shown in Figure 5a insert. The aggregation of the nanoparticles that being driven by the van der Waals attraction force should be the main reason for the poor transparency [26]. The transmittances at the same wavelength for PC/GNP-CA and $\mathrm{PC} / s$-GNP are even lower than that of PC/GNP, especially for $\mathrm{PC} / s$-GNP. This result can be explained as following. First, the un-denatured protein particles cannot improve the quality of GNP dispersion (Fig-

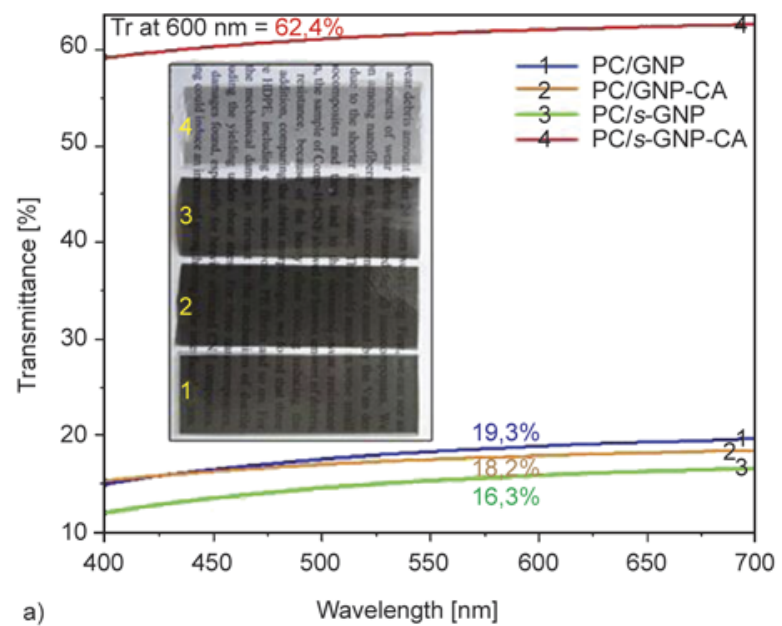

ure $4 b, 4 c)$; second, the big SPI particles themselves will further deteriorate the optical properties. In comparison, a dramatic increase in optical transparency

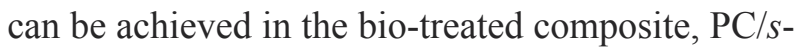
GNP-CA. The transmittance is up to $62 \%$ at $600 \mathrm{~nm}$ wavelength. The significantly improved transparency should be contributed by two different factors related to the denatured SPI: a good GNP dispersion and the reduction of GNP thickness (Figure 4d) result in the translucency of the composite film. The high light transmittance of the nanocomposite shows its high potential in solar energy conversion applications, such as solar cells.

It is well-known that dispersion of conductive nanofillers in a polymer matrix is a vital factor determining electrical properties of the resultant nanocomposites. It has been demonstrated that the $\mathrm{AC}$ conductivity $(\sigma)$ of a polymeric nanocomposite is a function of frequency when the filler loading is below the percolation threshold, and becomes frequency independent at a concentration level above the percolation threshold [27]. As shown in Figure 5b, the value of $\sigma$ is strongly dependent on the frequency for pure PC due to its insulating nature. Similar behavior has been observed for PC/GNP, PC/GNP-CA and $\mathrm{PC} / s$-GNP, indicating that the addition of pristine GNP, and the GNP treated by only CA or poorly denatured SPI all contribute little to improving the electrical conductivity mainly due to the poor filler dispersion. However, the bio-treated sample, $\mathrm{PC} / s$ GNP-CA, shows a conductive property (the frequency independent behavior) mainly due to the improved GNP dispersion. In particular, the value of $\sigma$ for PC/s-GNP-CA increases almost 11 orders of

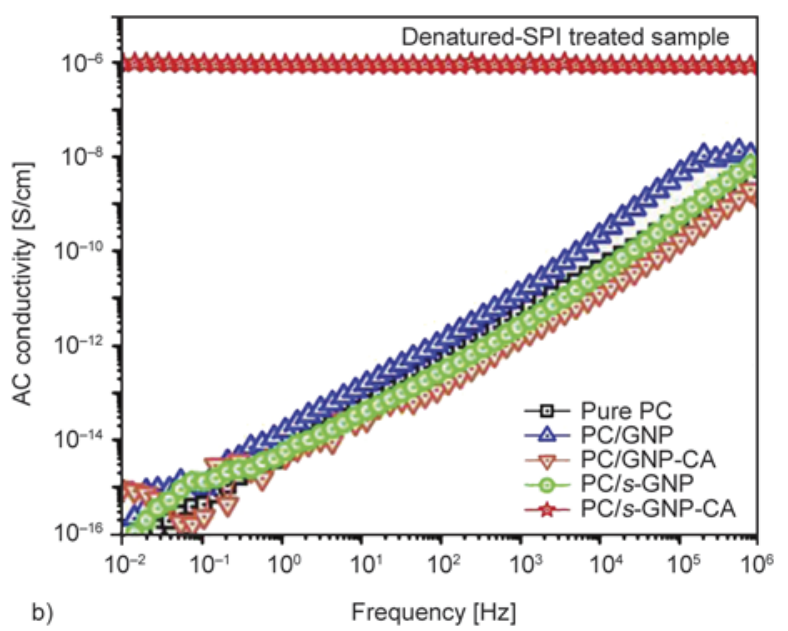

Figure 5. Properties of the nanocomposites with different GNPs: (a) optical properties as characterized by UV-vis spectroscopy; (b) AC conductive properties 
magnitude (at $10^{-2} \mathrm{~Hz}$ ) as compared to these of $\mathrm{PC} /$ GNP or the other two treated samples. With the same electrical conductivity improvement, the loading for $\mathrm{PC} / \mathrm{s}$-GNP-CA composite $(1 \mathrm{wt} \%)$ is lower than the values reported in other studies (usually above $2 \mathrm{wt} \%$ ) [28]. The good conductive and optical properties of the polymeric nanocomposite with GNP treated by the denatured SPI may make the nanocomposite very promising as flexible, transparent conductors, which are highly desired by flexible energy devices, such as flexible solar cells.

\section{Conclusions}

Soy protein was effectively denatured in a nonaqueous environment with a denaturing agent, cetyl alcohol. An effective biotreatment for nanofillers by the use of the denatured soy protein has been realized, for the first time to our knowledge, in an organic solvent. The studies demonstrate that soy protein denaturation led to homogenous nanoscale SPI particles (below $100 \mathrm{~nm}$ ) in the organic solvent with the aid of the denaturing agent. The nano-scaled denatured SPI become an effective bio-treatment agent for nanoparticles, such as GNPs. When nanocomposites were fabricated with the bio-treated GNPs, it was found that the denatured SPI was able to infiltrate between GNP layers to reduce the thickness of GNPs, at the same time to notably improve the filler dispersion in the nanocomposites. As one example of the application of the denatured SPI, a $\mathrm{PC} / s^{-}$ GNP-CA nanocomposite with good optical and electrical performances has also been shown in this study, which indicates that high-performance nanocomposites with the potential for photoelectric applications, such as polymer solar panels, can be fabricated based on the bio-treatment of nanoparticles via the denatured SPI. In a word, this study has laid foundation for development of a facile, cost-effective and environmentally friendly biotreatment of nanofillers in organic environment, which will remarkably extend the great potential of natural proteins for fabricating high-performance nanocomposites based on organic solution.

\section{Acknowledgements}

The authors appreciate the technical support from the Franceschi Microscopy \& Imaging Center at Washington State University. The authors also gratefully acknowledge the support from NSF 1029940.

\section{References}

[1] Liu T., Wang Y., Eyler A., Zhong W-H.: Synergistic effects of hybrid graphitic nanofillers on simultaneously enhanced wear and mechanical properties of polymer nanocomposites. European Polymer Journal, 55, 210-221 (2014).

DOI: $10.1016 /$ j.eurpolymj.2014.04.002

[2] Wildgoose G. G., Banks C. E., Leventis H. C., Compton R. G.: Chemically modified carbon nanotubes for use in electroanalysis. Microchimica Acta, 152, 187214 (2006). DOI: $10.1007 / \mathrm{s} 00604-005-0449-x$

[3] Lu J., Drzal L. T.: Microfibrillated cellulose/cellulose acetate composites: Effect of surface treatment. Journal of Polymer Science Part B: Polymer Physics, 48, 153161 (2010). DOI: $10.1002 /$ polb.21875

[4] Kunioka M., Ninomiya F., Funabashi M.: Biobased contents of organic fillers and polycaprolactone composites with cellulose fillers measured by accelerator mass spectrometry based on ASTM D6866. Journal of Polymers and the Environment, 15, 281-287 (2007). DOI: $10.1007 / \mathrm{s} 10924-007-0071-6$

[5] Fernandes E. M., Pires R. A., Mano J. F., Reis R. L.: Bionanocomposites from lignocellulosic resources: Properties, applications and future trends for their use in the biomedical field. Progress in Polymer Science, 38, 1415-1441 (2013).

DOI: 10.1016/j.progpolymsci.2013.05.013

[6] Kim J. T., Netravali A. N.: Physical properties of biodegradable films of soy protein concentrate/gelling agent blends. Macromolecular Materials and Engineering, 297, 176-183 (2012).

DOI: $10.1002 /$ mame.201100135

[7] Mari S. A., Köster S., Bippes C. A., Yildiz O., Kühlbrandt W., Muller D. J.: pH-induced conformational change of the $\beta$-barrel-forming protein OmpG reconstituted into native E. coli lipids. Journal of Molecular Biology, 396, 610-616 (2010).

DOI: $10.1016 /$ j.jmb.2009.12.034

[8] Ávila-Orta C. A., Cruz-Delgado V. J., Neira-Velázquez M. G., Hernández-Hernández E., Méndez-Padilla M. G., Medellín-Rodríguez F. J.: Surface modification of carbon nanotubes with ethylene glycol plasma. Carbon, 47, 1916-1921 (2009). DOI: $10.1016 /$ j.carbon.2009.02.033

[9] Numata M., Asai M., Kaneko K., Bae A-H., Hasegawa T., Sakurai K., Shinkai S.: Inclusion of cut and as-grown single-walled carbon nanotubes in the helical superstructure of schizophyllan and curdlan ( $\beta$-1,3-glucans). Journal of the American Chemical Society, 127, 58755884 (2005).

DOI: $10.1021 / \mathrm{ja} 044168 \mathrm{~m}$

[10] Shen J., Shi M., Yan B., Ma H., Li N., Hu Y., Ye M.: Covalent attaching protein to graphene oxide via diimide-activated amidation. Colloids and Surfaces B: Biointerfaces, 81, 434-438 (2010). DOI: $10.1016 /$ j.colsurfb.2010.07.035 
[11] Liu Y., Yu D., Zeng C., Miao Z., Dai L.: Biocompatible graphene oxide-based glucose biosensors. Langmuir, 26, 6158-6160 (2010).

DOI: $10.1021 / 1 \mathrm{a} 100886 \mathrm{x}$

[12] Zheng M., Jagota A., Semke E. D., Diner B. A., Mclean R. S., Lustig S. R., Richardson R. E., Tassi N. G.: DNAassisted dispersion and separation of carbon nanotubes. Nature Materials, 2, 338-342 (2003).

DOI: $10.1038 /$ nmat 877

[13] Zhang J., Jiang L., Zhu L., Jane J-L., Mungara P.: Morphology and properties of soy protein and polylactide blends. Biomacromolecules, 7, 1551-1561 (2006). DOI: $10.1021 / \mathrm{bm} 050888 \mathrm{p}$

[14] Liao Y., Farrell T. P., Guillen G. R., Li M., Temple J. A. T., Li X-G., Hoek E. M. V., Kaner R. B.: Highly dispersible polypyrrole nanospheres for advanced nanocomposite ultrafiltration membranes. Materials Horizons, 1, 58-64 (2014).

DOI: $10.1039 / \mathrm{C} 3 \mathrm{MH} 00049 \mathrm{D}$

[15] Liu D., Zhu C., Peng K., Guo Y., Chang P. R., Cao X.: Facile preparation of soy protein/poly(vinyl alcohol) blend fibers with high mechanical performance by wet-spinning. Industrial and Engineering Chemistry Research, 52, 6177-6181 (2013).

DOI: $10.1021 / \mathrm{ie} 400521 \mathrm{a}$

[16] Bhattacharyya S., Sinturel C., Salvetat J. P., Saboungi M-L.: Protein-functionalized carbon nanotube-polymer composites. Applied Physics Letters, 86, 113104/1113104/3 (2005).

DOI: $10.1063 / 1.1883725$

[17] Nepal D., Geckeler K. E.: Proteins and carbon nanotubes: Close encounter in water. Small, 3, 1259-1265 (2007). DOI: $10.1002 / \mathrm{smll} .200600511$

[18] Karajanagi S. S., Yang H., Asuri P., Sellitto E., Dordick J. S., Kane R. S.: Protein-assisted solubilization of single-walled carbon nanotubes. Langmuir, 22, 1392-1395 (2006). DOI: $10.1021 / 1 \mathrm{a} 0528201$

[19] Wu Y., Hudson J. S., Lu Q., Moore J. M., Mount A. S., Rao A. M., Alexov E., Ke P. C.: Coating single-walled carbon nanotubes with phospholipids. The Journal of Physical Chemistry B, 110, 2475-2478 (2006). DOI: $10.1021 / \mathrm{jp} 057252 \mathrm{c}$
[20] Bandyopadhyaya R., Nativ-Roth E., Regev O., Yerushalmi-Rozen R.: Stabilization of individual carbon nanotubes in aqueous solutions. Nano Letters, 2, 25-28 (2002). DOI: $10.1021 / \mathrm{nl} 1010065 \mathrm{f}$

[21] Ji J-Y., Lively B., Zhong W-H.: Soy protein-assisted dispersion of carbon nanotubes in a polymer matrix. Materials Express, 2, 76-82 (2012).

DOI: 10.1166/mex.2012.1055

[22] Zhang Y., Zhao W., Yang R., Ahmed M. A., Hua X., Zhang W., Zhang Y.: Preparation and functional properties of protein from heat-denatured soybean meal assisted by steam flash-explosion with dilute acid soaking. Journal of Food Engineering, 119, 56-64 (2013). DOI: 10.1016/j.jfoodeng.2013.05.008

[23] Li B., Olson E., Perugini A., Zhong W-H.: Simultaneous enhancements in damping and static dissipation capability of polyetherimide composites with organosilane surface modified graphene nanoplatelets. Polymer, 52, 5606-5614 (2011).

DOI: $10.1016 /$ j.polymer.2011.09.048

[24] Abbasi S., Carreau P. J., Derdouri A.: Flow induced orientation of multiwalled carbon nanotubes in polycarbonate nanocomposites: Rheology, conductivity and mechanical properties. Polymer, 51, 922-935 (2010). DOI: $10.1016 /$ j.polymer.2009.12.041

[25] Lively B., Zhong W-H.: An efficient quantified stereological macrodispersion analysis approach for determining microscale influences on nanocomposite material properties. Macromolecular Materials and Engineering, 298, 221-234 (2013).

DOI: $10.1002 / \mathrm{mame} .201200086$

[26] Lu C., Zu Y., Yam V. W-W.: Specific postcolumn detection method for HPLC assay of homocysteine based on aggregation of fluorosurfactant-capped gold nanoparticles. Analytical Chemistry, 79, 666-672 (2007). DOI: $10.1021 / \mathrm{ac} 061513 \mathrm{c}$

[27] Liu T., Wood W., Li B., Lively B., Zhong W-H.: Electrical and dielectric sensitivities to thermal processes in carbon nanofiber/high-density polyethylene composites. Science and Engineering of Composite Materials, 18, 51-60 (2011). DOI: $10.1515 / \mathrm{secm} .2011 .007$

[28] Li J., Vaisman L., Marom G., Kim J-K.: Br treated graphite nanoplatelets for improved electrical conductivity of polymer composites. Carbon, 45, 744-750 (2007).

DOI: $10.1016 /$ j.carbon.2006.11.031 